\title{
Sind die Gallengangstuberkel in der Leber das Resultat einer Ausscheidungstuberkulose?
}

\author{
Von \\ Dr. med. Mieczyslaw Lichtenstein, Warschau.
}

Über die Frage, wie entstehen die sogenannten Gallengangstuberkel, sind die Ansichten noch geteilt.

Simmonds hatte früher die Ansicht vertreten, dass der Prozess etwa in Analogie mit der tuberkulösen Peribronchitis, als Pericholangitis einsetze, und dass bei weiterem Wachstum der Tuberkel diese allmählich immer mehr auf die Wand der Gallengänge übergreifen, bis schliesslich ein Durchbruch dieser Massen in das Lumen hinein erfolgt. Der Prozess schreite also von aussen nach innen fort.

Demgegenüber ist Kotlar der Ansicht, dass zwischen den Tuberkeln und den Gallengängen eine primäre Beziehung nicht bestehe. Es kann aber durch ständige Vergrösserung eines Tuberkels allmählich auch die Wand eines Gallenganges ergriffen werden und schliesslich ein Durchbruch, also ebenfalls von aussen nach innen erfolgen. Auf die Ausführungen Kotlars werden wir weiter unten noch zurückkommen.

Nun hat aber Simmonds nenestens eine ganz andere Ansicht über die Entstehung des Gallengangstuberkel ausgesprochen. Er glaubt, dass sie einer A us scheidungs tuberkulose ihren Ursprung verdanken. Dass der Modus der Ausscheidungstuberkulose vorkommt, war ja schon länger, durch die Untersuchungen von $\mathrm{Hanau}$, Orth und von Meyer (für die Niere) nachgewiesen, und nach Simmonds wäre auch in der Tube dieser Modus nachzuweisen. Nach Simmonds entständen auch die Gallengangstuberkel so: Das primäre ist die Ausscheidung der Bazillen in der Galle; sie gelangen so in die Gallengänge, infizieren diese sekundär, und nun greift der Prozess vom 
Lumen des Gallenganges weiter über auf die Wand und seine Umgebung, also im Gegensatz zu der oben erwähnten Ansicht, ron innen nach aussen.

Im Gegensatz zu dieser neuen Auffassung Simmonds steht nun aber die ron Joest und Emshoff. Diese hatten bei 57 spontan tuberkulösen Tieren (Rindern und Schweinen) den Tuberkel. bazillengehalt der Galle geprüft, und 14 mal positive Befunde erhoben. Eine tuberkulöse Erkrankung der Gallenblase wie der grösseren Gallengänge bestand nicht. Joest und Emsh off erklären den Befund folgendermaassen: Die Bazillen müssen aus tuberkulösen Veränderungen der Leber stammen. Diese liessen sich ja auch meist makroskop isch nachweisen. Diese tuberkulösen Veränderungen müssen nach den Ausführungen zu "offen" sein. Eine Ausscheidung von Bazillen durch eine gesunde Drüse gibt es niclıt.

Es fragt sich nun: gibt es überhaupt die Möglichkeit, aus dem anatomischen und histologischen Verhalten diese Frage, zumal die der "Ausscheidungstuberkulose", zu beantworten. Simmonds hat diese Frage in seiner zweiten Arbeit erörtert und sagt (l. c. S. 156):

"Die erste Forderung wird die sein, dass man in den Sekretionskanälen Tuberkelbazillen zu einer Zeit nawhweisen kann, wenn noch keine nennenswerten Veränderungen an der Kanalwand nachweisbar sind, die zweite Forderung ist die, dass die histologischen Veränderungen mit Sicherheit auf einen von den zentralen auf die peripheren Wandschichten sich ausbreitenden Prozess hinweisen. Dort, wo man diesen beiden Forderungen gerecht werden kann, ist man wohl berechtigt, den angegebenen Infektionsweg vorauszusetzen."

Der histologische Befund an kleinsten Gallengangstuberkeln musste (bei Annahme einer Ausscheidungstuberkulose) nach Simmonds folgender sein:

„Da wird man bei Anlegung von Serienschnitten auf Stellen treffen, wo das Kanälchen noch unversehrt ist, im Innern desselben aber bereits Tuberkelbazillen in geringer Anzahl vorbanden sind. Man wird weiter Partien begegnen, in welchen die Kanalwandung von Rundzellen infiltriert ist, während das Epithel noch erhalten ist. Dann löst sich dieses $a b$, nekrotisiert, und jetzt erst zerfällt das Wandinfiltrat rasch unter der Einwirkung der Galle und führt zu Kavernenbildung, während sich sekundär im umgebenden Lebergewebe neue Tuberkelablagerungen bilden." (1. c. S. 157/158.)

Für eine Sonderstellung. der Gallengangstuberkel, daher vermutlich auch besondere Genese dieser Gebilde spräche auch nach Si m monds, die Erfahrung, „dass jene Gallengangstuberkel entweder gar nicht oder gleich in grösserer Zahl in der Leber vorhanden sind, dass man 
sie gelegentlich auch dann antrifft, wenn tuberkulöse Ablagerungen in der Leber nur spärlich vorhanden sind, dass man sie sehr häufig vermisst, wenn das Organ dicht von miliaren Knötchen durchsetzt ist. Um ein zufälliges Zusammenstossen von Tuberkeln und Gallenkanalwandung kann es sich also unmöglich handeln." (S. 157.)

Auf Anregung von Herrn Professor Kaufmann habe ich nachzuweisen versucht, ob sich bei genauer Untersuchung geeigneter Leber nicht Anhaltspunkte für den einen oder anderen der oben genannten Entstehungmodi beibringen liessen.

Zuerst habe ich eine Leber ron einem älteren, an Lungentuberkulose verstorbenen Mann untersucht. Die Lunge enthielt eine sehr grosse Anzahl makroskopisch sichtbarer Tuberkel, doch keine makroskopisch als solche erkennbaren Gallengangstuberkel.

Eine zweite Leber stammte von einer 65 jährigen, an einem inoperablen 'Tumor (Karzinom) verstorbenen Frau, die ausserdem tuberkulöse. Herde in der Lunge und in den Halslymphknoten hatte. Hier waren in der Leber sehr zahlreiche stecknadelkopf- bis erbsengrosse Tuberkel vorhanden.

Um dies gleich vorweg zu nehmen: Die histologische Untersuchung von zahlreichen Stücken erster Leber an Serienschnitten hat für die Frage kein irgendwie entscheidendes Resultat ergeben.

In der zweiten Leber konnte ich an Serienschnitten nirgends eine Andeutung für den Übertritt der Galle in die verkäste Masse feststellen. Ich konnte dagegen in einigen Leberstückchen deutlich Durchbrüche der verkästen Masse in grössere Gallengänge nachweisen. In Serienschnitten war erst der Gallengang intakt, die verkäste Masse von ihm getrennt; sie nähert sich $\mathrm{ihm}$, wie die nächsten Schnitte zeigen, allmählich bis schliesslich ein Durchbruch festzustellen ist. Trotz der deutlichen Kommunikation der verkästen Masse mit dem Gallengang, konnte in der rerkästen Masse kejne Spur von Galle nachgewiesen werden. Wir haben hier also einen Gallengangstuberkel, dem das makroskopische Charakteristikum der galligen Färbung fehlt.

Die histologische Untersuchung hat hier auch keinerlei Anhaltspunkte darüber ergeben, dass die Infektion primär vom Gallengang aus erfolgt sei, vielmehr müssen wir hier einen Durchbruch des Prozesses von aussen nach innen annehmen.

Eine dritte Leber stammte von einem 57 jährigen Arbeiter, bei dem die Sektion tuberkulöse Prozesse vorwiegend in den Lungen, ergeben hatte. Hier fanden sich in der Leber in grosser Anzahl neben kleinen, gewöhnlichen Tuberkeln stecknadelkopfgrosse und auch grössere gallig gefärbte Herde, die konzentrisch von einem weissen 
Ring umgeben waren. Die gallige Masse im Zentrum bildete zum Teil eine erweichte Masse, an wenigen Stellen fand sich auch das Zentrum dieser Herde als kleine $\mathrm{Höhle,} \mathrm{mit} \mathrm{gallig} \mathrm{gefürbter} \mathrm{Wand.}$

Bei der histologischen Untersuchung mehrerer solcher Gallengangstuberkel an Serienschnitten konnte ich nun nirgends mikroskopische Befunde erheben, wie sie Simmonds Desideraten entsprechen (s. o.). Ich habe nie in einem intakten Gallengang Tuberkelbazillen nachweisen können, auch habe ich nie, bei noch intaktem Epithelüberzug des Ganges, Infiltrate in der Wand beobachtet.

Hingegen wurden (an Serienschnitten) folgende histologische Befunde erhoben: Man findet mehrere, einzelne, teils in Scheiden kleinerer Gallengänge, teils im Gefässe oder im interlobulären Bindegewebe entwickelte Tuberkel, die allmählich grösser werden, verkäsen, konfluieren (Konglomerattuberkel).

Diese verkästen Massen nähern sich allmählich einem (in gewisser Entfernung verlaufenden) grösseren Gallengang. Dieser ist erst völlig intakt, der ihn umgebende bindegewebige Ring völlig erhalten. Auf den nächsten Schnitten sieht man nun, dass die Ent fernung zwischen dem intakten Gang und der Peripherie der Käseherde immer kleiner wird. Jetzt findet sich auch auf der dieser Käsemasse zugekehrten Seite eine Infiltration. In den nächsten Schnitten ist an dieser Stelle die Wand des Gallenganges zerstört: also eine Kommunikation von Käseherd und Gallengang. In den nächstfolgenden Schnitten werden die gleichen Befunde erhoben: mit dem Unterschied, dass die verkästen Massen den Gallengang nicht bloss an einer Seite, sondern ringsum umgeben. In den weiteren Schnitten verschwinden aber die tuberkulösen Bildungen um den Gang, und dieser lässt sich weiterhin, völlig intakt, verfolgen.

An der Durchbruchsstelle sieht man, dass der Gang (der bei sehwacher Vergrösserung, Leitz Obj. 3, Oc. I) ca. 1/4 des Gesichtsfeldes einnimmt, noch wohl erhaltene Auskleidung mit Zylinderepithel zeigt. In der $\mathbb{N}$ ähe der Durchbruchsstelle findet sich eine intensiv grünlichgelb gefärbte, mehr oder weniger scharf abgegrenzte gallige Masse. Sie ist am intensivsten gallig gefärbt auf der Höhe der Durchbruchstelle; nach den anderen Richtungen hin wird sie blasser gefärbt und verliert sich allmählich.

In der Höhle selbst findet man teils einzelne, teils miteinander noch im Zusammenhang stehende Gallengangsepithelien.

Die gallige Färbung ist, wie gesagt, auf der Höhe der Durchbruchsstelle am intensirsten. Aus dem Gallengang muss also Galle ausgeflossen sein, die das nekrotische Gewebe nun imbibiert hat. Diese Galle stand, wie ich mir vorstellen möchte, möglicherweise unter 
einem gewissen Druck: so wäre es zu erklären, dass die Epithelien des Gallenganges zum 'T'eil als zusammenhängende Massen sich gelöst haben, und von der Wand grösstenteils abgehoben, nur noch an einer Stelle ihr anhaften, teils aber auch ausserhalb der Durchbruchsstelle in der verkästen Masse liegen.

Die in der verkästen Masse gefundenen Epithelien brauchen aber nicht bloss einem einzigen Gallengange anzugehören, sondern sie können ron mehreren abstammen. Zuweilen von recht kleinen, in einen grösseren Gang mündenden. Man kann bisweilen in Serienschnitten einen solchen kleinen Gang über ein paar Schnitte verfolgen, bis er sich völlig in eine verkäste Masse verliert. Die einzelnen Gruppen von Epithelzellen liegen auch oft in einem Schnitte zu weit voneinander entfernt, als dass sie zu einem Gang gehören könnten.

Nimmt ein Gallengang einen etwas gewundenen Verlauf, so kann er, oberhalb oder unterhalb der Durchbruchstelle, wieder inmitten verkäster Massen zu liegen kommen. So können Bilder einer Pericholangitis vorgetäuscht werden. Solche Stellen sind mir aber nur in späteren Stadien begegnet, nie in Anfangsstadien.

Meine Befunde decken sich so vollkommen mit denen Kotlars, dass ich die Ausführungen dieses Autors hier wörtlich zitieren kann:

"In der Höhle jener Kavernen, in denen Gallenpigment erhalten war, bildete einen konstanten Befund das Vorbandensein von Gallengangsepithelien. Die Frage nach der Herkunft dieser Epithelmassen, welche von der Mehrzahl der Autoren für Epithelreste eines einzigen Gallenganges, dessen Lumen zur Bildung der Kaverne diente, gehalten werden, konnte nur mittelst der Serienschnittmethode gelöst werden. Auf diesem Wege gelang es mir oft zu rerfolgen, wie solche Epithelreste allmählich in mehrere verschieden grosse, in der Kavernenwand gelegene Gallengänge übergehen. Aus diesen Befunden geht hervor, dass diese Epithelreste keineswegs als Überreste eines einzigen Gallenganges, welcher für sich allein bei der Bildung der betreffenden Kaverne die Grundlage abgegeben hatte, angesehen werden könnten, vielmehr als Fragmente mehrerer Gallengänge, die in eine schon rorgebildete und allmählich sich vergrössernde Kaverne einbezogen werden, aufuufassen sein werden."

Sehen wir nun, was sich aus unseren histologischen Untersuchungen ergibt, so ist zu sagen: Wir haben nirgends an Gallengangstuberkeln, überhaupt an Tuberkeln in der Leber, solche Bilder gesehen, wie Simmonds Annahme einer A usscheidungstuberkulose und dadurch bedingten Infektion eines Gallenganges entsprächen. 
Die Frage, ob darum das Vorkommen einer Ausscheidungstuberkulose in der Leber und eine sich daran anschliessende Entstehung von sogenannten Gallengangstuberkeln überhaupt zu negieren sei, betrachten wir aber selbst damit noch nicht für erledigt.

A priori wäre die Möglichkeit eines solchen Entstehungsmodus wohl zu bejahen. Nur scheint uns, dass auch nach den Kriterien von Simmonds ein Beweis keineswegs mit Sicherheit erbracht sei, und in unseren Untersuchungen haben wir gerade die Simmondschen Kriterien eben nicht festgestellt. Aber gesetzt auch, man würde konstant die von Simmonds geforderten Befunde erheben: auch dann könnten die infizierenden Bazillen aus einem in der Nähe gelegenen „offenen" tuberkulösen Herd stammen.

Andererseits kann natürlich auch bei unserer Auffassung immer der Einwand gemacht werden: es ist zwar ein Durchbruch eines tuberkulösen Herdes in einen Gallengang erfolgt, aber die primäre Infektion kann trotzdem durch ausgeschiedene Bazillen erfolgt sein.

Können wir überhaupt anatomisch eine Entscheidung mit einiger Wahrscheinlichkeit treffen?

Simmonds sagt zwar, dass man die Gallengangstuberkel sehr häufig vermisst, wenn das ganze Organ dicht von miliaren Tuberkeln (also irgendwie metastatisch entstandenen) durchsetzt ist. Umgekehrt, dass man die Gallengangstuberkel, wenn überhaupt, gleich in grösserer Anzahl antrifft, gelegentlich auch dann, wenn tuberkulöse Ablagerungen (also doch wohl die gewöhnlichen Tuberkel) nur spärlich in der Leber vorbanden sind. Da also Simmonds die Gallengangstuberkel scharf von anderen Tuberkeln in der Leber trennt, und für jene einen besonderen Entstehungsmodus annimmt, so müsste man, wenn man schon ein sicheres Urteil abgeben wollte, fordern, dass man einmal in einer Leber nur Gallengangstuberkel anträfe, und keine anderen. Sobald aber die beiden, nach Simmonds genetisch verschiedenen Tuberkel da sind, kann man, je nach dem individuellen Standpunkt, jederzeit sagen: diese sind das primäre, jene das sekundäre oder umgekehrt.

Immerhin müsste künftighin genauer darauf geachtet werden, ob tatsächlich häufige Gallengangstuberkel reichlich in Lebern mit möglichst wenigen, anderen Tuberkeln rorkommen: das würde für die Annahme Sim monds sprechen.

Dann wäre festzustellen: wie verhalten sich die Leber überhaupt bei den verschiedenen Formen der Tuberkulose? Finden wir in Fällen, wo wir in anderen Organen, z. B. Niere, Prostata oder in den Tuben tuberkulöse Prozesse antreffen, die wir im Sinne einer Ausscheidungstuberkulose deuten dürfen, gerade in der Leber auch reich- 
lich Gallengangstuberkel, nach Simmonds also durch Ausscheidung von Bazillen entstandene Gebilde, oder nicht?

Und ferner: es müssten in grösserer Zahl Gallenblasen auf ihren Gehalt an Tuberkelbazillen geprüft und gleichzeitig genau histologisch untersucht werden, $o b$ in diesen Lebern überhaupt Tuberkel und etwa vorzugsweise Gallengangstuberkel sich finden. Vor allem müsste das in Fällen geschehen, wo nicht allzu ausgedehnte tuberkulöse Veränderungen in einem primären Herde, etwa der Lunge vorhanden wären.

Endlich kann vielleicht das Tierexperiment uns weitere Aufschlüsse über eine Infektion der Leber von der Galle aus liefern.

Vorerst müssen wir jedenfalls soviel sagen, dass durch anatomische und histologische Untersuchungen bisher noch kein sicherer Beweis für die Entstehung der Gallengangstuberkel durch eine Ausscheidungstuberkulose erbracht ist.

Herrn Geheimrat Professor Kaufmann bin ich für die Anregung zu dieser Arbeit und die freundliche Überlassung des Materials zu grossem Dank verpflichtet.

\section{Literatur.}

E. Kotlar, Über die Pathogenese der sogenannten Gallengangstuberkalose in der Leber des Menschen. Zeitschrift für Heilkunde, Bd. XV. 1894.

M. Simmonds, Tuberkulose der Gallengänge. Beiträge zur Statistik und Anatomie der Tuberkulose, Deutsches Archiv für klinische Medizin, Bd. XXVII, 1880.

M. Simmonds, Über Ausscheidungstuberkalose. Medizinisch-kritische Blätter, Hamburg 1910.

Joest-Emsh of $f$, Untersuchungen über den 'luberkelbazillengehalt der Galle bei tuberkulösen Tieren. Zeitschrift der Infektionskrankheiten, parasitäre Krankheiten und Hygiene der Haustiere, Bd. X, Heft 4, 1911. 